\title{
The Current Scenario of Pharmaceutical Research; 3d Tissue Engineering Considered as Eminent Technique
}

\author{
UmamaYezdani ${ }^{1}$, Mohammad Gayoor khan ${ }^{2 *}$, ZubiaZainab ${ }^{3}$, Mayur sadar ${ }^{4}$, Shivam \\ Choudghal $^{5}$, Damini Mishra ${ }^{6}$, Hari Baskar ${ }^{7}$ \\ ${ }^{I}$ Department of Pharmacy Practice, MRM college of Pharmacy, Hyderabad India \\ ${ }^{2}$ Department of Pharmacy, Truba Institute of Pharmacy, Bhopal India \\ ${ }^{3}$ Department of Pharmacy, MESCO college of Pharmacy, Hyderabad India \\ ${ }^{4}$ Department of Pharmacy, Anuradha College of Pharmacy, chikhali Maharashtra, India \\ ${ }^{5}$ Department of Pharmacy Practice, ISF College of Pharmacy, Punjab, india \\ ${ }^{6}$ Department of Pharmaceutics, Rameshwaram institute of technology and Management, India \\ ${ }^{7}$ Department of Pharmacy Practice, KMCH college of Pharmacy, Coimbatore Tamilnadu, India
}

*Corresponding Author: Mohammad Gayoor khan, student at Truba Institute of Pharmacy, Bhopal Madhya Pradesh, India. Email: rk981487@gmail.com

\begin{abstract}
Tissue engineering is an interdisciplinary field of pharmacy that utilizes cells, biomaterials, Biochemical it is part of Pharmaceutical engineering whereas Tissue engineering involves the use of a tissue scaffold for the formation of new type tissue for a clinical purpose. Which help in improving the methods of drug delivery and also drug Discovery in Pharmaceutical research for reducing force on central nervous for inflammatory response. This may also result in some neurodegenerative diseases. (Ex: Parkinson's, and Alzheimer's). This terminology is basically used in clinical and in vitro study includes Regenerative medicine, the drug discovery process, at molecular level analysis of cell. The major clinical need for this is for the treatment of degenerative tissues in this Review mainly focused on recent advancement of tissue engineering model that alter biophysiochemical properties for use in treatment of neurodegenerative disease. The continuing success of tissue engineering, and also the ultimate development of true human replacement parts, can grow from the convergence of engineering and basic analysis advances in tissue, matrix, growth factor, stem cell, and developmental biology, etc. apart from that extracellular matrix decellurizations as innovative and new scaffold treatment for disease. Chem $3 D$ software is using for drug design referred as modern methodology for computer aided Drug design.
\end{abstract}

Keywords: $3 D$ cell culture, decellularization, CAM/CAD technology, Neurodegenerative, Chem 3D software, Tissue engineering

Graphical Abstract: Tissues engineering is branch of Pharmaceutical Engineering Which help in improving the methods of drug delivery and also drug Discovery in Pharmaceutical research for reducing force on central nervous for inflammatory response. In Pharmaceutical research there are three types of methods used in tissues engineering for the treatment of neurodegenerative diseases. This is shown in figure 1.1;

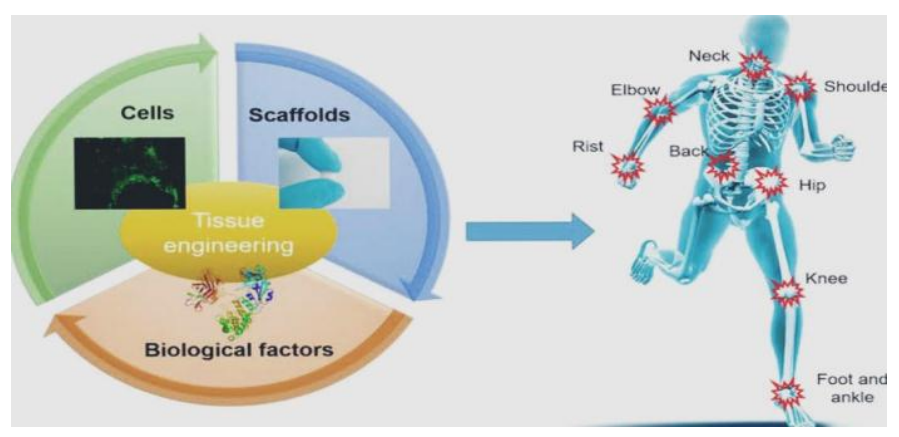

Figure No. 1.1 (a) Cell based Material (b) Animal Based Material Type (c) Engineered Material Type. 


\section{INTRODUCTION}

Tissue engineering is an interdisciplinary field that utilizes cells, biomaterials, biochemical (e.g., growth factors) and physical (e.g., mechanical loading) signals, as well as their combinations to generate tissue-like structures. First time tissues engineering was introduced in 1970s and in early 1980s which gave rise to modern tissue engineering the term is coined only 1987 at the ancient time first technique used in Egypt all of these treatments were based on non-living materials, and they provided some structure and function but were not like original tissues and organs ex: gold is used for teeth replacement, wood is used for toes, but after middle of the twentieth century, it was possible to replace an entire organ with an organ from a donor, today this is known as organ transplantational though this is an ultimate solution for organ failure but the need for organs always surpasses the number of available donated organs. The limitation of donor and rejection of the grafts by the immune system had shown the vitro method of tissue growing.

\section{History of Regenerative Medicine AND TISSUE ENGINEERING}

History shows that generation of new organ and changes in the tissues were the most primitive and imaginary concept of human. The revolution of the human race lea to the development of tissue engineering. In Egypt the use of this was made to restore body parts and also to develop new body parts and tissues for as inscribed in spells known as the "Pyramid Texts" (2375 BC). First dental prosthesis was made from gold in Egypt around $2500 \mathrm{BC}$. As the century passes the tissues engineering results have seen in positively due to higher result increase the confidence of person in believing regenerative medicine is useful technique for future aspects. In the field of research Extracellular matrix materials are commercially available and are used in reconstructive surgery, treatment of chronic wounds, and some orthopedic surgeries; as of January 2017 clinical studies were also going on to study how to repair heart tissues. While the regenerative medicine century wise shown in Pie chart figure no.1.2 and Table figure no. 1.3 respectively.

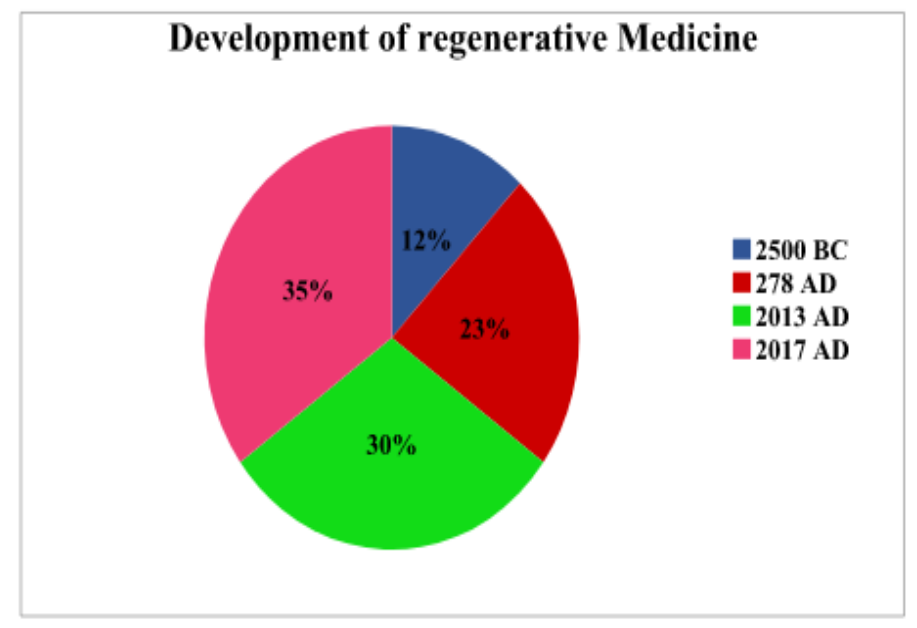

Figure No. 1.2 Pie Chart Year Wise Development in Tissue Engineering

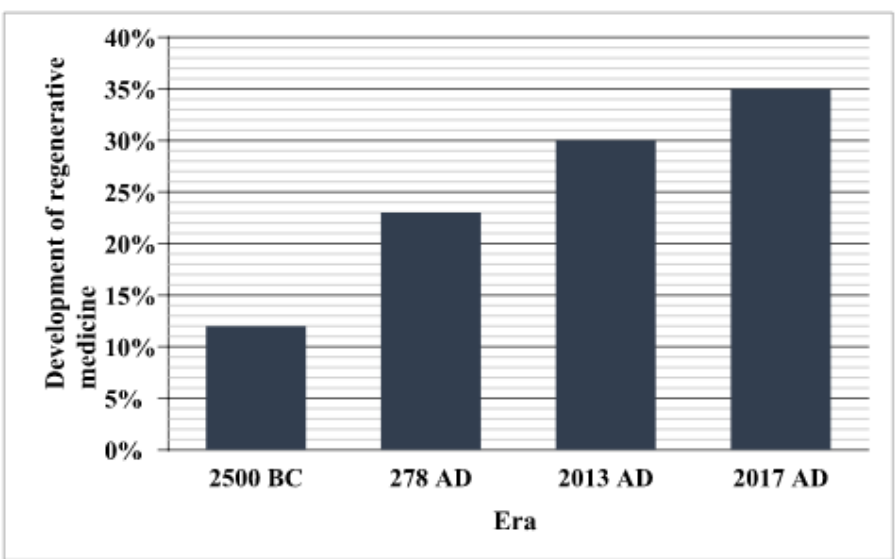

Figure No. 1.3 Graph Plot for Development of Regenerative Medicine 


\section{NEED FOR TISSUE ENGINEERING (Clinical Prospective)}

As per clinical prospectus tissue engineering and regenerative medicine are mainly needed as a result of our urge to treat defective tissues. The traditional medicine has several limitations in delivering solutions for many no. of health problems. Injuries and diseases are been treated by pharmaceuticals which are used for the treatment of many diseases, and prosthetic devices, organ transplantation are used in more severe and dreadful conditions. According to world Health Organization report tissue engineering and cell therapy market has been estimated in 2014 at about $\$ 17$ billion and is expected to grow up to $\$ 39$ billion by 2020 the CAGR (Compound Annual Growth rate increase $7 \%$ ). Where the orthopaedic market is followed by skin, organs, and tissues.

The skin was the very first tissue to be engineered; this can be due to the relatively simple structure of the tissue. Skin was also an important target for due tissue engineering due to its high demand specifically resulting from war burns. Skin damage can also cause various kinds of disability, which may further result in other serious infections and psychological damage to patients due to other environmental factors. These were few reasons about why skin was chosen to be the first engineered tissue. This technique can be applied on any tissue but the level of complexity is kept in notice. (Ex: the heart, kidneys, cornea, nervous tissues, liver, intestines, pancreas, lungs, bone, muscle, and so on). The major goal of tissue engineering is the development of accurate, effective and functional substitutes for damaged tissues and it can also fulfil the need for organ transplant.

\section{3D TISSUE EngINEERING TECHNIQUE}

A 3D cell culture is a form of artificially created surroundings within which biological cells are permissible to grow or interact with their surroundings in all the 3 dimensions. These three-dimensional cultures are typically fully grown in bioreactors, small capsules within which the cells will grow into spheroids or 3D cell colonies. In the 3D culture, we can control scaffold morphology, architecture, therefore, cells behave and respond as they would in vivo to stimuli. In living tissue, cells exist in 3D microenvironments with intricate cell-cell and cell-matrix interactions and complex transport dynamics for nutrients and cells. 3D spheroids more closely resemble in vivo tissue in terms of cellular communication and also the development of extracellular matrices. The basic classification of 3D culture methods can be classified into two types scaffold techniques and scaffold-free techniques. By using these techniques various diseases treated like Parkinson's and Central nervous neurodegenerative disease.

\section{BACKGROUND}

Neurodegenerative diseases and brain disorders have been some of the targets for pharmaceutical research. According to the World Health Organization, it is estimated 4 million cases of Alzheimer's disease in India. It has been estimated that the number of individuals suffering from Alzheimer's will increase to 14 million by 2040, while approx.400, 00 new diagnoses of Parkinson's occur every year in India. Neurodegenerative diseases will cost billions of dollars and also requires a profound medical care for years. The challenges faced at the time of treatment of Neurodegenerative disease is the Neuroinflammation by disorders this inflammatory response dividing force present behind these diseases. This results in the loss of neural cells and lack of functional recovery after disease onset.

When novel drug therapy came into the notice it was estimated that the neurodegenerative disorders can be cured very effectively in a very short duration of time. In this they try to find out various types of cures and find the most accurate among them and this also takes help of the tissue engineering to select a particular tissue and study on its active site etc, the therapies which are known till now and are regularly used are with the anti-inflammatory agents but that is not enough as it takes a longer time so further researchers are needed to be carried out for more effective treatment. This can be possible when the drug development and discovery improves so there is a need for the $3 \mathrm{D}$ techniques to come into frame for the development of pharmaceutical research.

\section{Role OF Microglia IN NEPHROPROTECTIVE DISEASE}

As there was no method that was so accurate to check the damage and aging of the cells of the brain and the neuron this method was employed 
very successfully and it mainly focused on increasing the immunity of the brain and to reduce aging and to product a positive effect on the brain cells.

Major functions of Neuroimmuniue cells:

- A sentinel function (this senses the changes in their environment constantly)

- Promotes neuronal well-being and normal operation

- A defence function necessary for responding to such changes and providing neuroprotection.

Microglia uses a defined armamentarium of genes to perform these tasks. In response to specific stimuli, or with neuroinflammation, microglia even has the capability to damage and kill neurons. Injury to neurons in various diseases and also results from disruption of the sentinel or its functions and dysregulation of the defence function and neuroinflammation. Immune systems have become targets of emerging pharmaceutical research, including that of the central nervous system (CNS). Unlike macrophages that serve as the immune cells for the rest of the body, microglia is the resident immune cells uniquely of the CNS and thus has many important functions in the brain. Unregulated and prolonged activation of these immune cells referred to as inactive Microglia are dynamically cells around the contracting process from the body, these processes are able to detect a minute change in their environment respond to foreign objects that require an immune response. The microglia cells also helps in the protection of the central nervous system and it function by sending the impulse about the unwanted effects going on in the cells. These soluble neuron-injury factors activate more microglial cells or serve to prolong the activated state of previously activated microglial cells and are very much effective in the treatment and need a proper therapy

\section{The Tissue Engineering Model}

Tissue engineering is an application of engineering and its major role is to treat or replace damaged tissues and organs. There are various models being organized and these models uses 3D matrices to culture cells, and to produce living tissues, which are same in morphology and function to that of normal ones. These can be used for studies of disease progression and propagation, drug discovery and compound screening, and they are also used in tissue repair and replacement. The use of organoids, 3D scaffolds, and decellularization techniques are the ways to create $3 \mathrm{D}$ materials for use in tissuespecific research.

By using the organoid system, it is highly possible to screen for potential compounds that will affect the diseased tissue with minimal side effects to healthy tissues. By using this we can even know the effect of various diseases on the brain tissues and cells. These types of models are used by the bio researchers in dreadful conditions or when no other method is being employed.

\section{The Role of 3D Printing in Tissue ENGINEERING PROCESS}

As pre clinical animal testing are very expensive it is not possible for everyone to carry out that process hence 3D engineering provides an helps us to do this in a very minimum cost and more effectively.

3D bioprinting can provide a high-throughput, reproducible alternative to these aforementioned methods for engineering neural tissues. While some of the more commonly used 3D printing methods, like fused deposition modeling, selective laser sintering and stereo lithography, it remains difficult to control the precise location when seeding multiple types of cells or localizing specific cues for controlling stem cell behavior. 3D bioplotting and microfluidic 3D printing methods allow for mixing of cells with bioinks, materials that can be printed while supporting cell culture, enabling the production of neural tissues.

\section{TARGETEd DRUG Delivery Methods FOR CENTRAL Nervous NeUrodegenerative DISEASEMANAGEMENT}

The big and major challenges to overcome neurodegenerative disease are the movement and absorption of drug from and across the blood brain barrier (BBB). The BBB is formed from a series of tight junctions mainly composed of endothelial cells, and astrocytes, macrophage, neuronal cells, pericytes, basal membranes, fibroblasts, and microglia. The major work of $\mathrm{BBB}$ is to protect the brain by restricting the passage of most substances from pathogens and disease into the CNS. 3D scaffolds are very effective in the treatment of neurodegenerative 
The Current Scenario of Pharmaceutical Research; 3d Tissue Engineering Considered as Eminent Technique

diseases as vehicles for the delivery of antiinflammatory drugs to sites of chronic inflammation following implantation to the brain. There are multiple ways in which these scaffolds may be utilized. Since the determined by the degradation rate of the 3D material.

The main two methodology used for Neurodegenerative using Drug Delivery system are respectively;

1. Diffusion-based methods

2. Immobilized drug delivery

\section{ANTI-INFLAMMATORY DRUGS}

NSAIDs are mainly known for their anti inflammatory, antipyretic and analgesic activity and hence constitute a major part of the study. Many of the compounds and other bioactive molecules shown good activity in in-vitro culture, microglia and other animal tissues and models. These same drugs which are shown to be effective in the treatment of Alzheimer's disease are even effect be in other neurodegenerative diseases which are shown in table figure no. 1.4 respectively:

Table 1.4. Type and status of the drugs (Anti-inflammatory Compounds) and its Effect as on Pro-inflammatory Mediators in the CNS.

\begin{tabular}{|c|c|c|}
\hline DRUGS & EFFECT & STATUS \\
\hline Marine algae & Reduces the pro-inflammatory mediators & Natural products/drugs \\
\hline Lycium Chinese & Suppress NO production in BV2. & Natural products/drugs \\
\cline { 1 - 2 } EHT & Reduces pro-inflammatory mediators in microglia. & Natural products/drugs \\
\cline { 1 - 1 } NSAID's & Reduces PGE2 levels by 50\%. & \multirow{2}{*}{ Approved drugs(allopathic) } \\
\hline Flurbiprofen & Reduces PGE2 levels by 50\%. & Approved drugs(allopathic) \\
\cline { 1 - 2 } Piroxicam & Reduces PGE2 levels by 50\%. & Approved drugs(allopathic) \\
\hline Indomethacin & Reduces PGE2 levels by 50\%. & Approved drugs(allopathic) \\
\hline Acetylsalicylic acid & Reduces PGE2 levels by 50\%. & Approved drugs(allopathic) \\
\hline Paracetamol & Reduce pro-inflammatory Intermediates in mice. & Phase II clinical trial \\
\hline PHPB &
\end{tabular}

A neuroprotective compound, potassium 2-(1hydroxypentyl)-benzoate (PHPB), shows to reduce levels of pro-inflammatory intermediates after LPS treatment in mice, and has shown effective in the treatment of Alzheimer's disease.

\section{Study Design}

This is the prospective study on randomly selected samples from 3D Tissue engineering technique for different methodology using treatment of Parkinson's, Central nervous neurodegenerative disease, Alzheimer's etc.

The Research will be conducted in Truba Institute, Bhopal, Madhya Pradesh, India.

\section{Methodology}

Induction of Neurodegenerative disease: Neurodegenerative are a group of conditions which mainly affects the neuron. As Neurons are the building blocks of the nervous system they cause abnormalities in brain and spinal cord. Example - Parkinson's disease.

On the Basis of Novel drug delivery Methods:

1. Diffusion-Based Method

2. Immobilized drug Delivery

\section{FUTURE DIRECTION}

3D engineering is one of the most important procedures for the drug screening and drug delivery for this it is very important to alter the chemical and biophysical properties of the materials to the appropriate tissue. Most recently this technique contains decellularization techniques to create 3D materials from cell extracellular matrix (ECM) materials complete with growth factors, proteins, and signaling molecules. In this decellularization technique matrix modification has also been included and the researches are being carried out for the same. Tissue specific method includes various components of cells and growth factors in their proper ratios. The advantage of this environment is they provide more efficient cell proliferation, attachment, and differentiation Retention of these and even help in the synthesis of the products used for the neuronal cells to develop and for the treatment of various diseases. Areas for future development of this technology are to provide proper vascularisation and they can even produce mimics of various barriers (ex: blood brain barrier) and can act as it for better treatment. These neural tissues could be printed tissues in arrays can help in proper screening of drug 
targets for the treatment of Alzheimer's disease to provide accurate tissues to be treated.

\section{CONCLUSION}

In short the use of regenerative methods and other tissue engineering techniques helps and ensures the development of new organ can be used as an important disciple in the fields of pharmacology, pharmaceutics and other fields of medicine. It can also be used in drug screening and in the development of drug delivery tools, regenerative methods can be used for the developing of the missing organs in mal nourished children and even in the development of new organ that has been completely destroyed.3D engineering is one of the most important method which can be successfully employed in the proper drug delivery in the body(novel drug delivery) to ensure the proper use of drug and dose in the body of the diseased persons and can even be counted as an important aspect in between drug and disease.

\section{ACKNOWLEDGMENT}

First of all I would like to Thank my Co- Author's Ms. Umama Yezdani (Department of clinical and Pharmacy Practice) MRM college of Pharmacy Hyderabad, India, Mr. Hari Bhaskar, Ms. Vinesha Ravi, Mr.Mulla Vasim akram, Mr. Ayush kumar. The authors are thankful to Dr. Rajesh Singh Pawar, PhD. Principal Truba Institute of Pharmacy for ceaseless encouragement during the study.

\section{REFERENCES}

[1] Gayoor Khan, Umama Yezdani, Rohit Verma, Raqshan Jabeen, Pradeep Sintha. Detection of Phlebovirus by using qualitative Real time (RT) - PCR and application of silver nanoparticles to control it. World J Pharm Pharm Sci.2018; 7(11) :936-52.

[2] Mohd.Gayoor Khan. The Novel Drug Delivery System. World J Pharm Pharm Sci.2017;6(7) :477-487.

[3] Unama Yezdani, Mohd. Gayoor Khan, Fazal Khan, Arvind Verma, Nilesh Kushwah, Rohit Verma. The Drug Targeting in Alzheimer's or Applications \& it's Hazards. World J PharmPharm Sci.2017;7(11):1532-1549.

[4] Dr.H.S Chandel., Sharad P.Panday, Arvind Dangi, Ashish chaurasia, Mohd.Gayoor khan et al. Development of Targeted Drug delivery. International Journal of research methodology Ijrm.Human., 2017 Vol. 1 (2): 30-34.

[5] Kushwah Nilesh, Yezdani Umama, Mohammad Gayoor khan, Manish kushwah, Kumar Ayush.
The Fundamental of Novel Drug Delivery System; Methodology, Role of Nanotechnology; Nanoparticles in Pharmaceutical Research. International Journal of Emerging Technologies and Innovative Research (www.jetir.org), ISSN:2349-5162, Vol.6, Issue 6, page no.140146, June-2019. DOI: http://doi.one/10.1729/ Journal.21510

[6] Mohd. Gayoor Khan, Nilesh Kushwaha, Fazal Khan, Vipul Patel. Microencapsulation. International Journal of research methodology Ijrm.Human., 2017 Vol. 1 (2):35-42.

[7] Umama Yezdani, Mohd. Gayoor Khan, Nilesh Kushwah, Arvind Verma,Fazal Khan. Application of Nanotechnology in Diagnosis and treatment of various disease and it's future advances in medicine. World J Pharm Pharm Sci.2018;7(11):1611-1633.

[8] Principles of Tissue Engineering - 4th Edition Editors: Robert Lanza Robert Langer Joseph. 45-76.

[9] Introduction to Tissue Engineering: Applications and 3D tissue engineering originally published: 2014 Author: Ravi Birla, 210-212.

[10] Tissue Engineering: Principles and Practices by John P. Fisher (Editor), Antonios G. Mikos (Editor), Joseph D. Bronzino (Editor), Donald R. Peterson (Editor), 87-93.

[11] Vascularization: Regenerative Medicine and Tissue Engineering Editor: Eric M. Brey. 457466.

[12] Culture of Cells for Tissue Engineering by Gordana Vunjak-Novakovic (Author), R. Ian Freshney (Author) page no. 673-682.

[13] Methods of Tissue Engineering Editors: Anthony Atala, Robert Lanza Page no.67-73.

[14] 14.DesRochers T.M., Suter L., Roth A., Kaplan D.L. Bioengineered 3D human kidney tissue, a platform for the determination of nephrotoxicity. PLoS One. 2013; 8: e59219. [PMC free article] [PubMed] [Google Scholar].

[15] Wang Y., Wei Y.T., Zu Z.H., Ju R.K., Guo M.Y., Wang X.M. Combination of hyaluronic acid hydrogel scaffold and PLGA microspheres for supporting survival of neural stem cells. Pharm Res. 2011; 28:1406-1414. [PubMed] [Google Scholar].

[16] Liu B., Hong J.S. Role of microglia in inflammation-mediated neurodegenerative diseases: mechanisms and strategies for therapeutic intervention. J Pharmacol Exp Ther. 2003; 304:1-7. [PubMed] [Google Scholar].

[17] Nimmerjahn A., Kirchhoff F., Helmchen F. Resting microglial cells are highly dynamic surveillants of brain parenchyma in vivo. Science. 2005; 308:1314-1318. [PubMed] [Google Scholar]. 
The Current Scenario of Pharmaceutical Research; 3d Tissue Engineering Considered as Eminent Technique

[18] Cugola F., Fernandes I., Russo F.B., Freitas B.C., Dias J.L., Guimarães K.P. The Brazilian Zika virus strain causes birth defects in experimental models. Nature. 2016; 534:267271. [PMC free article] [PubMed] [Google Scholar].

[19] Discher D.E., Janmey P., Wang Y.L. Tissue cells feel and respond to the stiffness of their substrate. Science. 2005; 310:1139-1143. [PubMed] [Google Scholar].

[20] Day R.O., Graham G.G. Non-steroidal antiinflammatory drugs (NSAIDs) BJM. 2013; 346: f3195. [PubMed] [Google Scholar].

[21] Ricciotti E., FitzGerald G.A. Prostaglandins and inflammation. Arterioscler Thromb Vasc Biol. 2011; 31:986-1000. [PMC free article] [PubMed] [Google Scholar].

Citation: Mohammad Gayoor khan, et al, The Current Scenario of Pharmaceutical Research; $3 d$ Tissue Engineering Considered as Eminent Technique. ARC Journal of Public Health and Community Medicine.2019; 4(2):16-22. DOI: dx.doi. org/ 10.20431/2456-0596.0402003.

Copyright: (C) 2019 Authors. This is an open-access article distributed under the terms of the Creative Commons Attribution License, which permits unrestricted use, distribution, and reproduction in any medium, provided the original author and source are credited. 